\title{
Das skalare, durch einen rechteckigen Impuls in einem homogenen medium erzeugte Potential
}

(Erste Naherungsrechnung)

\section{A. BeLluigi}

Wir wollen vorerst das Problem der Erregung des Erdbodens mit Impuls-Strömen grundlich untersuchen, um die Messung der "LueckenPotentiale ", (praktische Anwendung des Elflex), durchfuhren zu konnen. Die Autoren, die sich in der Vergangenheit mit dieser Methode beschäftigt haben, weisen zwar auf die Anwendung der Impuls-Ströme hin olne sie jedoch in ihren Theorien überhaupt $\mathrm{zu}$ berucksichtigen. Dies bedeutet gleich von Anfang an eine schwerwiegende Unzulanglichkeit, da dadurch die "Periodizität der Lücken " ausfallt.

Es seien: $\Gamma$ die Ladungsdichte, $\Phi$ das skalare Potential, $\mu$ die magnetische Permeabilitat, $k$ die Dielektrizitätskonstante, $\sigma$ die Leitfähigkeit.

Dies vorausgestellt, erfüllt die Ladungsdichte $(\Gamma)$, wie wir bereits gesehen haben, in einem homogenen Boden, folgende Gleichung:

$$
\frac{\partial \Gamma}{\partial t}+4 \pi \alpha \Gamma=0
$$

wobei $\alpha=\sigma / k, \quad 1 / \alpha=\tau$,

und somit:

$$
\Gamma=\gamma e^{-4 \pi a t}=\gamma e^{-4 \pi t / \tau}
$$

mit der Ladungsdichte $\gamma$ fur $t=0$; für $t=\tau$ ist $\Gamma=\gamma / 2,718$.

Andererseits genügt das "skalare Potential " folgender Gleichung:

$$
\Delta \Phi-\mu k \frac{\partial^{2} \Phi}{\partial t^{2}}-4 \pi \sigma \mu \frac{\partial \Phi}{\partial t}=-\frac{4 \pi}{k} \Gamma
$$

die infolge Gl. [2] und wenn man die Beziehung $v-1 / / \mu k$ (Signal- 
Geschiwndigkeit) berucksichtigen will, folgende Form (für $t>0$ ) annimmt:

$$
\Delta \Phi-\frac{1}{v^{2}} \frac{\partial^{2} \Phi}{\partial t^{2}}-\frac{4 \pi \alpha}{v^{2}} \frac{\partial \Psi}{\partial t}=-\frac{4 \pi}{k} \gamma e^{-4 \pi a t}
$$

Setzt. man:

$$
\Phi=\psi e^{-2 \pi \alpha t}
$$

so verwandelt sich Gl. [3'] in folgende:

$$
\Delta \psi-\frac{1}{v^{2}} \frac{\partial^{2} \psi}{\partial t^{2}}+\frac{4 \pi^{2} \alpha^{2}}{v^{2}} \psi--\frac{4 \pi}{k} \gamma e^{-2 \pi a t}
$$

Wenn man annimmt, das $(\alpha=\sigma / k)$ genügend klein ist, sodass die Grössen $\left(\alpha^{2}\right)$-ter Ordnung vernachlässigt werden konnen, so reduziert sich Gl. [5] auf nachstehende Beziehung:

$$
\Delta \psi-\frac{1}{v^{2}} \frac{\partial^{2} \psi}{\partial t^{2}}=-\frac{4 \pi}{k} \gamma e^{-9 \pi r t}
$$

die dieseble Form der "Wellengleichung ":

$$
\Delta_{\psi} \quad \frac{1}{v^{2}} \frac{\tau^{2} \psi}{\partial t^{2}}=-4 \pi f
$$

besitzt und aus der man die Losung mit Hilfe der "verzogerten Potentiale " erhält.

Wir heben sofort hervor, dass es nicht immer gestattet ist, die Grossen $\left(a^{2}\right)$-ter Ordnung $\mathrm{zu}$ vernachlässigen. In einer zweiten Näherungsrechnung wird hiervon Rechnung getragen werden.

Im Falle eines unendlich ausgedehnten homogenen Mediums nimmt die Losung folgende Form (Formel von Kirchhoff) an:

$$
\psi=\iint_{-\infty}^{+\infty} \varrho^{-1}[f] \underset{(t-v / \varrho)}{d \xi d \eta d \zeta}
$$

Der weggelassene Flächenintegral-Summand ist in identischer Weise gleich Null, was man erkennt, wenn die Fläche $\mathrm{S}$ vom Sender des einzelnen z. B. recheckigen Impulses so weit entfernt ist, dass zur Zeit $(t)$ noch kein Punkt derselben erreicht worden ist; das Symbol [ ] bezeichnet "verzogerte Werte".

Die Entfernung:

$$
\varrho=\left[(x-\xi)^{2}+(y-\eta)^{2}+(z-\zeta)^{2}\right]^{1 / 2}
$$

ausserdem muss $(t)$ in $(f)$ durch $(t=\varrho / v)$ ersetzt werden. 
Nehmen wir nun eine "punktförmige Elektrode" im Nullpunkt an, die in Zeitpunkt $(t=0)$ eine momentane Ladung $(q)$ liefert. Dies bedeutet, dass die Ladungsdichte gleich:

$$
\gamma=\varrho \cdot \delta(\xi) \delta(\eta) \delta(\zeta)
$$

gesetzt wird, wobei $\delta(\xi)$ die Funktion von Dimc bezeichnet, welche bekanntlich uberall gleich Null und für $\xi=0$ gleich unendlich ist, sodass:

$$
\int_{-\infty}^{+\infty} f(\xi) \delta(\xi) d \xi=f(0)
$$

$\iiint G(x, y, z ; \xi, \eta, \zeta) \delta(\xi) \delta(\eta) \delta(\zeta) d \xi d \eta d \zeta=\varrho(x, y, 0 ; 0,0,0)$

Da ferner, für $t<0$, das Feld gleich Null ist, muss man für $(f)$ in Gl. [6] folgenden Ausdruck setzen:

$$
\left.\begin{array}{l}
f=0 \quad, \quad \text { für } t<0 \\
f=-\frac{q}{r k} \delta(\xi) \delta(\eta) \delta(\zeta) e^{-2 \pi a t} .
\end{array}\right\}
$$

Mit diesem Wert von $(f)$, infolge der vorherigen Definition der Funktion von " Dirac », liefert Gl. [7] sofort:

$$
\begin{aligned}
& \psi=0, \quad \text { für } t<r / v \quad ; \quad r^{2}\left(x^{2}+y^{2}+z^{2}\right) \\
& \psi=\frac{q}{r k} \exp [-2 \pi a(t-r / v)], \text { für } t>r / v .
\end{aligned}
$$

Aus den Gl. [10] und [4] erhält man unmittelbar für die $\Phi$ :

$$
\begin{aligned}
& \Phi=0 \quad, \quad \text { für } t<r / v \\
& \Phi=\frac{a}{r \bar{i}} \exp [-2 \pi \alpha(2 t-r / v)] \quad, \text { für } t>r / v .
\end{aligned}
$$

Wenn der Impuls lingegen im Zeitpunkt $(\tau)$ anstatt im Zeitpunkt $(t=0)$ erzeugt wird, dann genügt es in die Gl. [11], $(t-\tau)$ an Stelle von $(t)$ zu setzen und man erhält:

$$
\left.\begin{array}{l}
\Phi=0, \quad \text { für } t-\tau<r / v \\
\Phi=\frac{q}{r k} \exp [-2 \pi a(2 t-2 \tau-r / v)], \text { für } t-\tau>r / v .
\end{array}\right\}
$$

Nehmen wir an, dass der rechseckige Impuls die Dauer $(T)$ habe und dass somit im Zeitintervall $(0<\tau<T)$ vom Strom $J=Q / T$ die Ladung $(Q)$ geliefert werde. Um dic diesem Zustande entsprechenden Formeln zu erhalten, genügt es offensichtlich die Wirkungen der ein- 
zelnen Impulse zu integrieren. Man gelangt auf diese Weise zum folgenden Ausdruck für das Potential $(\varphi)$ :

$$
\varphi=\int_{0}^{T} \Phi(\tau) d \tau
$$

wobei $(\Phi)$ durch Gl. [11'] gegeben ist und an Stelle von Gl. [9] die Beziehung $J=Q / T$ eingesetzt wurde.

Auf Grund der doppelten Definition von $(\Phi)$ siehe Gl. [11'] muss man drei Falle unterscheiden:

a) $t<r / v$, d. h. vor Ankunft des Impulses in der Entfernung $(r)$.

b) $r / v<t<r / v+T$ d. h. die der Ankunft des Impuls-Beginnes folgende jedoch seinem Ende vorstehende Augenblicke.

c) $t>r / v+T$ d. h. Augenblicke die der Ankunft des gesamten Impulses folgen.

Im Falle a) ist die $\Phi(\tau)=0$ im gesamten Intervalle $(0<\tau<T)$ und somit ist $(\varphi=0)$.

In Falle b) ist $\Phi(\tau)=0$ fuer $\tau>t-r / v$ und verschieden von Null fuer $(0<\tau<t-r / v<T)$ sodass die $(\varphi)$ siehe Gl. [11'] und [12] aus folgender Beziehung berechnet wird:

$$
\varphi=\frac{J}{r k} \int_{0}^{t-r / v} \exp [-2 \pi \alpha(2 t-2 \tau-r / v)] d \tau
$$

Im Falle c) ist $\Phi(\tau) \neq 0$ im gesamten Intervalle $(0, T)$ und wird infolgedessen die Integration auf das gesamte Intervall ausgedehnt. Die $(\varphi)$ wird somit durch folgende Beziehung:

$$
\varphi=\frac{J}{r k} \int_{0}^{T} \exp [-2 \pi \alpha(2 t-2 \tau-r / v)] d \tau
$$

berechnet. Nach Durchführung der angegebenen Integrationen, gelangt man für die $(\varphi)$ zu folgenden Ausdrücken:

$$
\begin{aligned}
\varphi & =0, \text { für } t<r / v \\
\varphi & =-\frac{J}{2 \pi \sigma r}\left[e^{-2 \pi \alpha r / v}-e^{-2 \pi \alpha(2 t-r / v)}\right]= \\
& =\frac{J}{2 \pi \sigma r} \sin h 2 \pi \alpha(t-r / v) e^{-2 \pi \alpha t}, \text { für } \frac{r}{v}<t<\frac{r}{v}+T \\
\varphi & =\frac{J}{2 \pi \sigma r} \sin h 2 \pi \alpha T \cdot e^{-2 \pi \alpha(2 t-T-r / v)} . \text { für } t>\frac{r}{v}+T .
\end{aligned}
$$

Gap Potential 
Man beachte, dass für einen festen Wert von $(r)$ die $(\Phi)$ den Wert $(0)$ bis zum Zeitpunkt $r / v$ (Ankunft des Impuls Beginnes) annimmt, bis zum Zeitpunkt $(r / v+T)$ (Ankunft des Impuls-Endes) bis zum Höchstwert:

$$
\varphi_{m}=\frac{J}{2 \pi \sigma} \sin h 2 \pi \alpha T e^{-2 \pi \alpha T} \cdot \frac{e^{-2 \pi \alpha r / v}}{r}
$$

anwächst und nachher rapid bis Null abnimmt.

Für $t=T+r / v$ nimmt das "Gap-Potential" folgende Form an:

$$
\begin{aligned}
& \varphi=\frac{J}{2 \pi \sigma r} \sin h 2 \pi \alpha T e^{-2 \pi \alpha}\left[2 T+2 \frac{r}{v}-T-\frac{r}{v}\right] \\
& \varphi=\frac{J}{2 \pi \sigma r} \sin h 2 \pi \alpha T e^{-2 \pi \alpha(T-r / v)}, \text { oder fur } t=T+\frac{r}{v}+r \\
& \varphi=\frac{J}{2 \pi \sigma r} \sin h 2 \pi \alpha T e^{-2 \pi \alpha}\left[T+2 \tau-\frac{r}{v}\right]
\end{aligned}
$$

\section{ABSTRACT}

The theory exposed in this paper gives an approximative evaluation of the "electric scalar potential " in a low conductivity undefinite homogeneous medium, generated by an electrode which supplies a " unit current pulse ", with velocity of propagation $v$.

The case of a "square" pulse of duration $T$ is examined, obviously it is sufficient to integrate the effects of each pulse.

Therefore the "direct scalar potential" and the "gap potential" are approximatively evaluated.

\section{RIASSUNTO}

La teoria qui data comporta una valutazione approssimativa del "potenziale elettrico scalare" in un mezzo indefinito omogeneo a bassa conduttività, generato da un elettrodo che fornisce un "impulso unitario" di corrente, propagantesi con velocità $v$.

$\grave{E}$ esaminato anche il caso di un "impulso rettangolare" di corrente di durata $T$, integrando gli effetti d'ogni singolo impulso unitario. Pertanto si calcola approssimativamente sia il potenziale diretto che il gap potential. 